\title{
Macht und Ohnmacht der Parlamente - 10. Passauer Symposium zum Parlamentarismus am 31. März und 1. April 2011
}

„Does parliament matter?“ Ob und inwieweit Parlamente ihre Stellung als demokratische Kerninstitution halten können, ist in der politikwissenschaftlichen Betrachtung nicht neu. Trotzdem oder gerade weil die Diskussion darüber nicht an wissenschaftlichem Interesse und politischer Brisanz verloren hat, war es Thema des 10. Passauer Symposiums zum Parlamentarismus. Die Deutsche Vereinigung für Parlamentsfragen, die Akademie für Politische Bildung Tutzing und die Universität Passau luden vom 31. März bis 1. April 2011 unter Leitung von Heinrich Oberreuter nach Passau, um sowohl Bestandsaufnahmen als auch zukünftige Entwicklungslinien von Parlamenten in der Bundesrepublik Deutschland, den USA und verschiedenen europäischen Staaten zu diskutieren.

Die Leitfrage nach der Marginalisierung von Parlamenten stellte Oberreuter an den Beginn des Symposiums. Der Leiter der Akademie für Politische Bildung Tutzing und bis 2010 Professor an der Universität Passau stellte dabei besonders den diagnostizierten Positionsverlust im politischen Entscheidungsprozess in den Mittelpunkt seines Vortrages. Dabei besprach er drei zentrale Elemente: (1) Die These von der „post-parlamentarischen Demokratie“, die geprägt ist von Interessengruppen und anderen privaten Akteuren, die nicht nur Einfluss auf den politischen Output haben, sondern diesen gezielt mitgestalten - unter Stärkung der Exekutive und Marginalisierung der Legislative. Den Parlamenten bliebe nur der rein symbolische Akt, die aktive Mitgestaltung könnten Abgeordnete aus Gründen der Komplexität aber auch aus Mangel an „intellektueller Kompetenz“ nicht mehr leisten. Dies führe zur Aushöhlung zweier unverzichtbarer Bestandteile parlamentarischer Demokratie: Transparenz und Partizipation.

(2) Unter dem Stichwort der Entparlamentarisierung durch eine zunehmende Europäisierung setzte sich Oberreuter kritisch mit dem exekutiv geprägten Entscheidungs- und Informationssystem auseinander, das es dem Bundestag erschwere, die Regierung zu kontrollieren und europäische Politik effektiv mitzugestalten. Besonders die Verbindlichkeit europäischer Rechtsrahmen schränke die Gestaltungs- und Aushandlungsmöglichkeiten nationaler Parlamente ein.

(3) Die Mediendemokratie schließlich erschwere es den Parlamenten und den einzelnen Abgeordneten, Aushandlungs- und Entscheidungsprozesse zu führen und zu transportieren. Die Parlamente stünden folglich im Spannungsfeld zwischen tradierten Abläufen und Ritualen einerseits, der Schnelllebigkeit und thematischen Selektion der Medien andererseits. Abschließend konstatierte Oberreuter jedoch, dass sie keineswegs so marginalisiert sind, wie es die These der post-parlamentarischen Demokratie versucht glauben zu machen. Parlamente bleiben die Kerninstitutionen der Demokratie, sie stellen die Responsivität und Legitimität des politischen Systems sicher. Parlamente stehen unter Druck, doch die Regierung ist auf die Rückbindung an ihre Mehrheit angewiesen, und diese darf sich, genau wie die Opposition, nicht von exekutiv geprägten Handlungsmustern entmündigen lassen.

Im Vortrag des Bundestagspräsidenten Norbert Lammert zum Thema „Parlament und Partizipation in der Mediendemokratie“ diente die Frage des Journalisten Bernd Ulrich „Was ist bloß mit uns los?" als Leitmotiv. Als Kritiker der Medienlandschaft und deren politischer Berichterstattung - besonders der öffentlich-rechtlichen Rundfunk- und Fernsehanstalten - trug Lammert nicht nur bei der Konstituierenden Sitzung des 17. Deutschen 
Bundestages zur Auseinandersetzung mit diesem Themenbereich bei. Die Vermittlung von Politik durch die Medien stellte Lammert jedoch grundlegend in Frage: Die gesteigerte Geschwindigkeit, in der die Medien politische Themen auswählen und darstellen, führte zu einer mangelnden Gründlichkeit politischer Berichterstattung. Zudem sei es zu einer explosionsartigen Vermehrung der Medien- und Lobbyvertreter in der Berliner Republik gekommen, die den Unterhaltungswert einer Nachricht vor die Recherche stellten. Scheinplebiszite und Scheindebatten des "Politainments" schadeten der ausgewogenen Abwägung von Sachverhalten und ließen die Öffentlichkeitsfunktion zu „reinem Populismus verkommen", was sich auch in einem Vertrauensverlust der Politik widerspiegele. Die Darstellung parlamentarischer Zusammenhänge sowie die Aushandlung politischer Entscheidungen würden zunehmend medial begleitet. Lammert plädiert deshalb dafür, sich der Schnelllebigkeit der Mediendemokratie nicht zu unterwerfen: „Ein Parlament, das sich ernst nimmt, müsse sich beständig gegen diese Entwicklung zur Wehr setzen."

Einen weiteren Diskussionspunkt setzte Werner Patzelt (TU Dresden) in seinem Vortrag „Konstruktion und Dekonstruktion. Zur Phänomenologie von Parlamentsmacht“. Ein Exkurs durch die historischen Entwicklungslinien, in dem Patzelt sich vorwiegend auf die aristotelische Vier-Ursachen-Lehre bezog, mündete in der Frage, was Parlamente machtlos macht. Patzelt konstatierte dabei fünf wesentliche Faktoren: (1) die Unattraktivität politischer Ämter, die sich in einem mangelnden Sozialprestige, in Zeitdruck und unangemessener Bezahlung widerspiegelten; (2) die zentrale Steuerung der Kandidatenaufstellung führe zudem nicht nur zu einer Machtlosigkeit der Wähler, sondern auch der Parteibasen und damit zu einem Schwund innerparteilicher Demokratie; (3) die mangelnde personelle Ausstattung unterbinde die Möglichkeit der thematischen Selbstbeschäftigung und - mit Verweis auf Oberreuters Kritik an Abgeordneten - deren intellektuelle Kompetenz; (4) der Entzug des Rechts auf Regierungskritik führe zur Verstummung einzelner Abgeordneter, die nur durch medial gesetzte Zeichen Aufmerksamkeit erreichen könnten. Doch auch der Zugang zu den Massenmedien sei für Regierungen und deren Parlamentsmehrheit privilegiert. Das „Ende der Diskussion“ sei dabei vorprogrammiert; (5) die Beschneidung formaler Machtmöglichkeiten, die sich beispielsweise in der Änderung von Tagesordnungen widerspiegelte. So würde aus einer Gesinnungsfrage eine Machtfrage. Da diese fünf Prozesse verdeckt verlaufen, warnte Patzelt abschließend vor einer „Verlandtagung des Bundestages".

Der ehemalige Präsident des Bundesverfassungsgerichts Hans-Jürgen Papier schloss sich seinem Vorredner an. Am Beispiel des verfassungsrechtlich fragwürdigen Atom-Moratoriums der Bundesregierung beschrieb er die Entmachtung des Bundestages, die keinesfalls mit den Regeln des Grundgesetzes konform sei. De jure könnte die Bedeutung des Parlaments größer nicht sein, de facto werde sie jedoch zunehmend ausgehöhlt. Die schleichende Entparlamentarisierung zeige sich, so Papier, nicht nur in der Kompetenzverlagerung auf die Europäische Union oder durch die Länder an den Bund, sondern auch in der mangelnden Transparenz politischer Entscheidungsprozesse, die von außerparlamentarischer Vorund Mitarbeit geprägt seien. Der deutsche Exekutivföderalismus schränke die Mitgestaltungsmöglichkeiten der Landesparlamente ein, sowohl auf Bundes- als auch auf europäischer Ebene. Seinen Vortrag „Entparlamentarisierung und Selbstverantwortung der Parlamente" beendete Papier mit dem Vorschlag, in die Landesverfassungen einen Europaartikel einzubauen, der die Vertreter der Regierungen im Bundesrat in solchen Fragen an das Votum ihrer Parlamente bindet. 
Plädierte Patzelt noch für offene Vorwahlen der Abgeordneten nach US-Prinzip, um die Kompetenz der gewählten Vertreter zu sichern, sprach sich Suzanne S. Schüttemeyer (Martin-Luther-Universität Halle-Wittenberg) deutlich für die Ochsentour der zukünftigen Parlamentarier durch die Parteien aus. Nur dies könne sicherstellen, dass die Volksvertreter ihr Handwerk von Grund auf lernen, vor allem das mühsame Geschäft, das demokratische Willensbildung und Entscheidung letztlich ausmacht, nämlich Mehrheiten zu schaffen. In ihrem Vortrag zum Thema „Abgeordnete: Repräsentanten oder Rädchen im Getriebe?“ beleuchtete sie das Kräftefeld, in dem Bundestagsabgeordnete stehen, die sich im Wahlkreis als responsiv gegenüber Wünschen und Interessen von Wählern erweisen müssen, in ihren Fraktionen aber in arbeitsteilig und hierarchisch strukturierte Abläufe eingebunden sind. Ihr weitgehender Verzicht auf Allzuständigkeit und eigenständige Rechte des einzelnen ist aber nicht Resultat eines Führungsdiktats, sondern spiegelt „vor allem die parlamentarischen Traditionen und Erfahrungen sowie das Selbstverständnis der Bundestagsabgeordneten“. Dass deren Effizienzorientierung sogar zum Repräsentationserfolg beitragen kann, illustrierte Schüttemeyer mit ersten Ergebnissen aus dem DFG-geförderten Projekt „Citizens and Representatives" (CITREP) der Universitäten Halle, Stuttgart und Bordeaux: 50 Prozent der befragten Deutschen attestierten den Berliner Parlamentariern, dass sie kompetente Arbeit im Bundestag leisten, 23 Prozent schätzten diese sogar als gut oder sehr gut ein.

Im Anschluss gaben Ellen Bos (Andrássy Universität Budapest), Roland Sturm (FriedrichAlexander-Universität Erlangen-Nürnberg), Josef Braml (Deutsche Gesellschaft für Auswärtige Politik) und Stefan Köppl (Akademie für Politische Bildung Tutzing) Einblick in aktuelle Entwicklungen verschiedener parlamentarischer Systeme. So stellte Sturm mit Blick auf Großbritannien fest, dass das Westminster-Modell mehr Teilhabe durch Opposition und Bürger zulasse, als von außen der Eindruck entstehe. Die seit 2010 in Großbritannien regierende konservativ-liberale Koalition habe eine Stärkung der politischen Rolle des Parlaments und der Kommunen angekündigt. Auch wenn die öffentliche Debatte zurzeit von Fragen der Sparpolitik beherrscht wird, sollte der stille Verfassungswandel in seinen Wirkungen nicht unterschätzt werden. Das Zusammenspiel von Repräsentantenhaus und Präsident in den USA war Thema von Josef Braml. Anschaulich diagnostizierte er einen schleichenden Kompetenzverlust politischer Institutionen und schloss sein Referat mit einer im Anschluss viel diskutierten These, dass wirklich handlungsfähig in den USA nur noch die Notenbank sei, die die Macht sowohl der Abgeordneten als auch des Präsidenten beschneide beziehungsweise diese sogar gänzlich einschränke. Eine ähnlich düstere Beschreibung des Parlamentarismus lieferte Ellen Bos, die sich mit den Transformationsprozessen einiger mittel- und osteuropäischer Staaten beschäftigte. Sowohl für die Ukraine als auch für Ungarn stellte sie erhebliche Defizite im demokratischen Prozess des Parlamentarismus fest. Politik würde zum Geschäft; Korruption und Vetternwirtschaft halten sich in der Ukraine hartnäckig. Der weitverbreiteten Annahme, dass in Italien - mit „Bunga-Bunga“ und Berlusconis Machtpolitik - ein Verfall des Parlamentarismus eingetreten sei, trat Stefan Köppl entgegen. Die Politik habe mit einem intensiv gepflegten, wenn auch turbulenten Parlaments- und Parteileben viel mehr zu bieten.

Den Abschluss des zweitägigen Symposiums zum Parlamentarismus lieferte Martin Sebaldt (Universität Regensburg), der sich - in Anlehnung an sein 2009 erschienenes Buch - mit der Macht und Leistungsfähigkeit von 23 etablierten Parlamenten befasste. Hier wurde besonders deutlich, wie sehr einerseits das Funktionsspektrum von Parlamenten differiert und andererseits das jeweilige parlamentarische Funktionsprofil durch bestimmte 
Kontextvariablen - Regierungssystem, Parteienlandschaft, politisches Vetospielergefüge und andere - beeinflusst wird. Des Weiteren zeigte sein Vortrag, welche unterschiedlichen parlamentarischen Leistungsbilanzen - zum Beispiel Gewährleistung von Regierungsstabilität, effektive Gesetzgebung, Haushaltsdisziplin, Bürgervertrauen - aus der jeweiligen Machtausstattung resultieren können.

Nach dem zweitägigen Symposium bleibt, trotz all der kritischen Anmerkungen und der Rede vom Ende des Parlamentarismus, festzustellen: „Parliaments do matter“. Sie garantieren die demokratische Legitimation eines politischen Systems und können durch ihre vielfältigen Kontrollmöglichkeiten einer expansiven Politik der Exekutive entgegenwirken. Sie stellen die Kommunikation und Responsivität zwischen Regierenden und Regierten sicher, wobei - und da waren sich alle Experten einig - eine wichtige Rolle auch dem einzelnen Abgeordneten und seinem Verständnis als Parlamentarier zukommt.

Romy Höhne 